\title{
Hyperconjugation in Carbocations, a BLW Study with DFT approximation
}

\author{
Zakaria Alamiddine ${ }^{\dagger}$ and Stéphane Humbel* \\ Centrale Marseille, Aix Marseille Université, CNRS, iSm2 UMR 7313, Marseille, France
}

Edited by:

Miquel Solà, University of Girona,

Spain

Reviewed by:

Israel Fernandez, Universidad

Complutense de Madrid, Spain

Eduard Matito, University of Girona,

Spain

*Correspondence:

Stéphane Humbel, Centrale

Marseille, Aix Marseille Université,

CNRS, iSm2 UMR 7313,

13397 Marseille, France

e-mail: stephane.humbel@

univ-amu.fr

${ }^{\dagger}$ Present address:

Zakaria Alamiddine, Université de

Nantes, CNRS, CEISAM, BP 92208,

44322 Nantes, France
The geometry of ethyl cation is discussed, and the hyperconjugation effect in carbocations is evaluated at the B3LYP/6-311G(d) level. The Block Localized Wavefunction (BLW) method is used for all evaluations of the hyperconjugation, considered as the energy gained by the delocalization onto the $\mathrm{C}^{+}$atom. This energy is defined as the energy difference between the delocalized (standard) calculation, where the electrons are freely delocalized, and a localized form where the positive charge sits on the carbon center. It is evaluated for 18 carbocations, including conjugated systems. In these cases we were particularly interested in the additional stabilization brought by hyperconjugative effects. Among other effects, the $\beta$-silicon effect is computed. Hyperconjugation amounts in several cases to an energy similar to conjugation effects.

Keywords: carbocation, valence bond, silicon, hyperconjugation, conjugation

\section{INTRODUCTION}

Carbocations' stabilization by hyperconjugation is one of the cornerstones of chemistry, and has received a considerable attention, particularly in educational, organic, and theoretical literature (Hehre, 1975). They are involved in numerous reactions, whenever an anionic chemical group leaves a carbon atom, as it is the case in $\mathrm{S}_{\mathrm{N}} 1$ reaction for instance, or by positively charged species attachment (White et al., 1999). Olah et al. have boosted their experimental study with exceptionally strong acids (Olah, 1993, 2001). However, computational studies are essential for the evaluation of the energetics at work (Lambert and Ciro, 1996; Müller et al., 2005). The recent review by Aue (2011) made a special emphasis on the study of their stability, plus a presentation of carbocations of practical interest, for instance in biological systems.

Carbocations stability is a key in numerous reaction mechanisms, particularly near transition states, where bond breaking/forming processes modify the electronic density of a species. Thus, their stabilization occurs frequently in systems that can be distorted from their equilibrium geometry. Their stability relies particularly on charge delocalization over the whole chemical species, and this can be attained via conjugation and hyperconjugation (Müller et al., 2005; Hadzic et al., 2011; Newhouse and Baran, 2011; Emanuelsson et al., 2013; Zimmerman and Weinhold, 2013). Even when it is small in magnitude, hyperconjugation can determine reactivity, and is of primary importance (Cieplak, 1999; Ingold and DiLabio, 2006; Braïda et al., 2009; Fernandez et al., 2013).

The conformation plays an important role, and sometimes it can be used to switch the delocalization off (deconjugated bond), and evaluate its effects by difference with the conjugated conformation (Wiberg et al., 1990; Gobbi and Frenking, 1994).

Conjugation involves an interaction between $\pi$ orbitals. It reputedly implies large stabilization energy (or resonance energy) and the effect extends across several bonds (Milian-Medina and Gierschner, 2012). Here, in the carbocation case, the charge delocalization corresponds to the interaction between the empty $\pi$ orbital of the carbocation center, and at least one filled $\pi$ orbital expanded on neighboring atoms (Scheme 1A) (Alabugin et al., 2011). We shall use the allyl cation as a model system to evaluate such stabilization. The hyperconjugation (Scheme 1B) involves filled $\mathrm{CH}$ orbitals, which are in principle lower in energy. Because orbitals interact better if they are close in energy, the effect is in principle larger for conjugation than for hyperconjugation.

As stated above in the allyl cation, a deconjugation by rotation around a CC bond (Scheme 2) can give an estimation of the resonance energy. However, this gives an underestimation of the energy because hyperconjugative effects lower the rotated structure (Mo, 2004). The value of the resonance energy in allyls has been the subject of some debates, which chiefly concerned the allyl anion (Mo et al., 1996; Mo and Peyerimhoff, 1998; Barbour and Karty, 2004; Linares et al., 2008). As far as the cation is concerned, there are less discrepancies among the authors although electronic correlation is significant and some variations are encountered. We shall retain that, with an Orbital Deletion Procedure (ODP) (Mo, 2006) the resonance energy in the allyl cation was evaluated to $36.6 \mathrm{kcal} / \mathrm{mol}$ at the HF level, and to 48.8 with B3LYP/6-311+G(d,p) level using the "Block Localized Wavefunction" (BLW) approach (Mo et al., 2007). These correspond to "Adiabatic Resonance Energies" (ARE), which means that geometrical parameters are relaxed in 


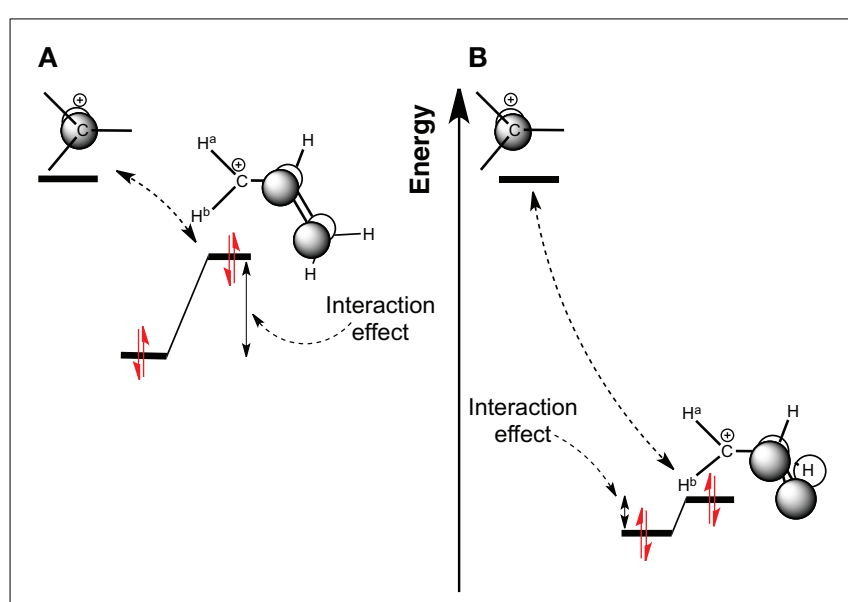

SCHEME 1 | Molecular Orbital diagram of a carbocation 2p orbital, interacting with (A) a $\pi-\mathrm{CC}$ bonding orbital; (B) a $\pi-\mathrm{CH}$ bonding orbital.

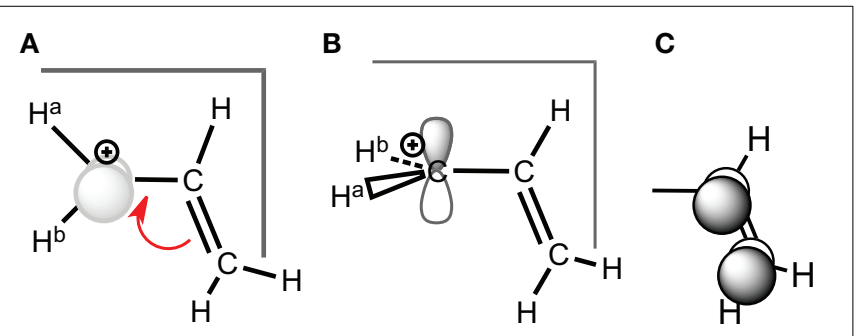

SCHEME 2 | (A) Conjugated carbocation for the allyl cation; (B) deconjugated case; (C) corresponding conjugated $\pi$-orbital.

the localized calculation (Figure 1). The later value is close to the value of $50 \mathrm{kcal} / \mathrm{mol}$, obtained with a corrected Hydride Ion Affinities procedure (HIA) (Aue, 2011). It is also close to the value obtained with our Lewis-based Valence Bond BOND scheme, $55 \mathrm{kcal} / \mathrm{mol}$ (Linares et al., 2006), although our value is to be considered as a Vertical Resonance Energy (VRE) because the geometry of the localized structure is constrained to that of the delocalized system.

As reminded above, hyperconjugation is frequently considered as a (small) second order conjugation, which is justified by the lower energy of $\mathrm{CH}$ bonding orbitals compared to $\pi$ (Scheme 1). This is particularly true in neutral systems, and the interaction can be small in these cases. However, there are evidences that hyperconjugation can be large, and even of similar magnitude as conjugation (Daudey et al., 1980; Mullins, 2012; Wu and Schleyer, 2013). Large hyperconjugation effects (in silicon substituted species) are reported to lead to very significant rate enhancements (up to $10^{12}$ times larger) (Lambert and Chelius, 1990; Creary and Kochly, 2009). They also have been isolated and an X-ray structure is even available. ${ }^{1}$

Several approaches are being used to describe the conjugation and hyperconjugation effects. We reminded in the introduction different publications using isodesmic reactions, based on

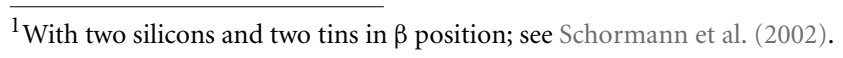

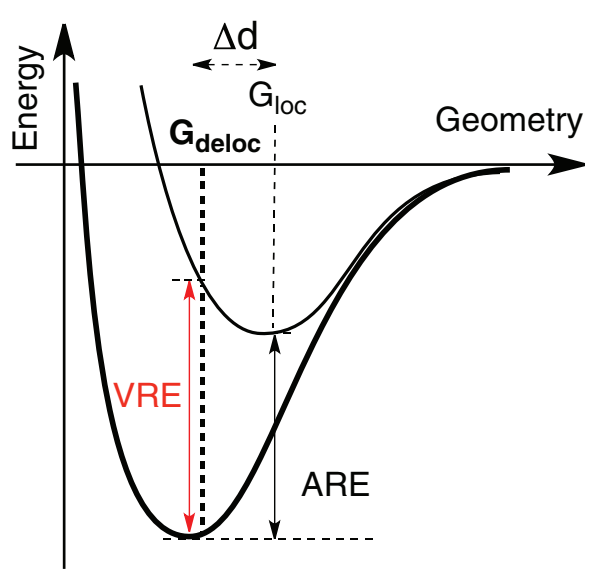

FIGURE 1 | Vertical (VRE) and Adiabatic (ARE) Resonance Energy. In bold is the energy curve of the delocalized wave function, while in plain is the localized. VRE and ARE differ by the geometry used to compute the localized wave function. The optimum geometry with the delocalized wave function is $G_{\text {deloc, }}$ and $G_{\text {loc }}$ for the localized one.

hydride abstraction. Hyperconjugation in neutral systems has also been described by a large panel of methods such as the Energy Decomposition Analysis (EDA) (Fernandez and Frenking, 2006; Feixas et al., 2011; Mo et al., 2011), Valence Bond (VB) (Shaik and Hiberty, 2008; Braïda et al., 2009; Havenith and Van Lenthe, 2009) or the already mentioned BLW (Bickelhaupt and Baerends, 2003; Mo et al., 2004a,b; Mo and Schleyer, 2006; Wu et al., 2012), and the well-known Natural Bond Orbital analysis (NBO) (Weinhold, 2003; Glendening et al., 2012).

There is also a rich literature on silicon substituted carbocations, and cross method evaluations have been recently published on these systems (Fernandez and Frenking, 2007; Dabbagh et al., 2012, 2013). Still on carbocations, Schleyer et al. very recently (Wu and Schleyer, 2013), showed large hyperconjugation effects in various strained systems. However, for simple carbocations, which are our subject here, there have been fewer studies. Particularly, the values published by Mo (2006) with the BLW method at the HF level have not been updated with a care for electronic correlation.

Such an evaluation at the correlated level is certainly desired, and this is an objective of the present paper. We evaluated the energetics of hyperconjugation at the B3LYP/6-311G(d) level, which includes some correlation effects. We used the BLW approach in all the cases. Despite some discussions on its apparent basis set dependency (Mo et al., 2010; Zielinski et al., 2010) this type of calculation is becoming a standard for such an evaluation (Steinmann et al., 2011; Wu et al., 2012; Fernandez et al., 2013). Our BLW results shall update and extend the values published previously at the uncorrelated level (Mo, 2006). We expect that, as it was the case for conjugation, correlated value for hyperconjugation will be somehow larger than Hartree-Fock.

The paper is organized as follows. In the computational considerations, we first define our levels of calculations, programs we used, and we write a short memo on the way we used the "BLW" approach in the specific case of carbocations. We then turn our 
attention to the conformations of the ethyl cation as a model of all the hyperconjugated cations. The results and discussion part is divided into three subsections. The first one deals with the hyperconjugation in the ethyl cation. The second extends to secondary and tertiary carbocations, with methyl substituents and silicon $\beta$-effects. In the last part, we added a conjugated link between the $\mathrm{C}^{+}$atom and the substituent (e.g., a C\#C triple bond). We evaluated here the incremental stabilization due to hyperconjugation in already conjugated species.

\section{COMPUTATIONAL CONSIDERATIONS}

The computations of the ethyl cation displayed in Table 1 were done with Gaussian 03 (Frisch et al., 2004). The three methods (HF, B3LYP, and CCSD) were used for the geometrical optimization with two basis sets, Pople's 6-311G(d) (Krishnan et al., 1980) and Dunning's cc-pvQZ (McLean and Chandler, 1980). DFT calculations are not very sensitive to the size of the basis set, but CCSD is much more basis set dependant. For the B3LYP calculations we used the default implementation of Gamess, with the original VWN5 correlation functional ${ }^{2}$ rather than the defaults Gaussian's implementation (Vosko et al., 1980; Lee et al., 1988; Becke, 1993). ${ }^{3}$ As it is also the default in Gamess, 6D orbitals were used throughout. As the basis set dependency was small only the 6-311G(d) results are discussed here. The results obtained with the cc-pvQZ basis set are given in the supplementary materials.

For all the BLW calculations, we used a version of Gamess that was modified by Mo to implement the BLW method (Mo and Peyerimhoff, 1998; Mo et al., 2000; Cembran et al., 2009). This implementation permits to re-optimize the geometry of the cations with the localization constraints. This feature was used to compute the geometrical effects of the localization: we obtained the $\mathrm{CC}^{+}$bond lengthening, and its impact on the resonance energy (hence we obtained both VREs and AREs). However, the ARE will be of little use here. We rather discuss the vertical energies because they can concern directly and unequivocally reactive intermediates in their genuine geometry. Geometrical variations upon localization/delocalization $(\Delta \mathrm{d})$ are discussed though.

With BLW, we used there the standard 6-311G(d) basis set, with no diffuses on heavy atoms and no polarizations on hydrogens. These restrictions intend to preserve the localized calculations' meaning. Diffuse orbitals on first neighbors of the carbocation, as well as $\pi$ orbitals on $\mathrm{H}$ atoms could bring some confusion on the validity of the localization constraints (Galbraith et al., 2013). In these calculations we oriented the systems in such a way that the $\pi$ system is along the $\mathrm{z}$ axis, and two blocks are defined for the localized calculations. One contains zero electrons (it is empty), and is defined over the $\mathrm{p}_{z}, \mathrm{~d}_{x z}, \mathrm{~d}_{y z}$ atomic orbitals of the $\mathrm{C}^{+}$site. This block ensures an appropriate localization of the positive charge. The other block contains all the electrons and is defined over all the remaining orbitals. For the delocalized calculations, we removed the (empty) block, and added the $\mathrm{p}_{z}, \mathrm{~d}_{x z}, \mathrm{~d}_{y z}$ atomic orbitals to the other block, so delocalization is now allowed.

\footnotetext{
${ }^{2}$ Gamess (Version R2, 25 March 2010) from Iowa State University (Schmidt et al., 1993).

${ }^{3}$ The following keywords were used in Gaussian inputs: BV5LYP $\operatorname{iop}(3 / 76=1000002000) \operatorname{iop}(3 / 77=0720008000) \operatorname{iop}(3 / 78=0810010000)$.
}

Table 1 | Energetics and key geometrical parameters of the ethyl cation in the conformations of Figure 2 ( $\Delta \mathrm{E}$ in $\mathrm{kcal} / \mathrm{mol}, \mathrm{d}_{\mathrm{CC}^{+}}$in $\AA$, $L_{\mathrm{CCH}}$ in ${ }^{\circ}$.

\begin{tabular}{|c|c|c|c|}
\hline Conformation & (1a) & (1b) & (1c) \\
\hline \multicolumn{4}{|l|}{$6-311 G(d)$} \\
\hline \multicolumn{4}{|l|}{ HF } \\
\hline$\Delta \mathrm{E}$ & 0.0 & -0.8 & -0.6 \\
\hline$d_{C^{+}}$ & 1.438 & 1.427 & 1.373 \\
\hline$\angle \mathrm{CCH}$ & 115 (107) & 95 & 58 \\
\hline \multicolumn{4}{|l|}{ CCSD } \\
\hline$\Delta \mathrm{E}$ & 0.0 & $x$ & -5.0 \\
\hline$d_{\mathrm{CC}^{+}}$ & 1.425 & $x$ & 1.386 \\
\hline$L_{\mathrm{CCH}}$ & 117 (107) & $x$ & 58 \\
\hline \multicolumn{4}{|l|}{ B3LYP } \\
\hline$\Delta \mathrm{E}$ & 0.0 & $x$ & -3.0 \\
\hline$d_{C^{+}}$ & 1.412 & $x$ & 1.380 \\
\hline$L_{\mathrm{CCH}}$ & $118(108)$ & $x$ & 58 \\
\hline
\end{tabular}

For the conformation (1a), the second angle (added in parenthesis) concerns the $\mathrm{CCH}$ angle for the out of plane hydrogens. 1a

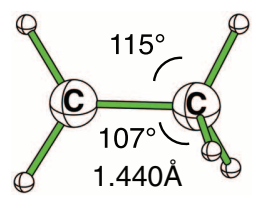

$1 \mathrm{c}$

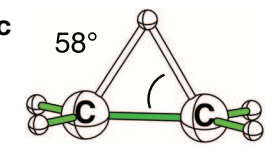

$1.373 \AA$

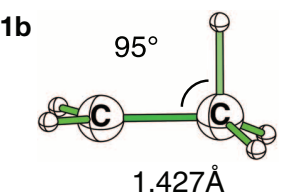

$1.427 \AA$

FIGURE 2 | HF/6-311G(d) optimized geometries for the three conformations of the ethyl cation.

The analysis of the BLW results concerns both energies and difference between electronic densities. For these densities we used two "cube" files generated by Gaussian 03. One has densities obtained with the orbitals of the localized calculation. The second uses the delocalized orbitals. ${ }^{4}$ The density differences at each point of the grid defined in the cube files were drawn with the VMD freeware. ${ }^{5}$ We refer to these drawings as Electronic Densities Difference maps (EDD maps). They indicate the flux of electron density (gain/loss) when localization constraints are relaxed.

\section{ETHYL CARBOCATION: ON THE CS GEOMETRY}

It is noteworthy that in the ethyl cation, which is the smallest system useful to describe and evaluate the hyperconjugation effects, the conformation with an hyperconjugation from $\sigma-\mathrm{CH}$ bonding

\footnotetext{
${ }^{4}$ Orbitals are given as "cards" in the Gaussian input files. Of course, they were not re-optimized.

${ }^{5} \mathrm{VMD}$ was developed by the Theoretical and Computational Biophysics Group in the Beckman Institute for Advanced Science and Technology at the University of Illinois at Urbana-Champaign (Humphrey et al., 1996).
} 


\section{A}

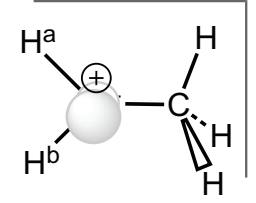

B
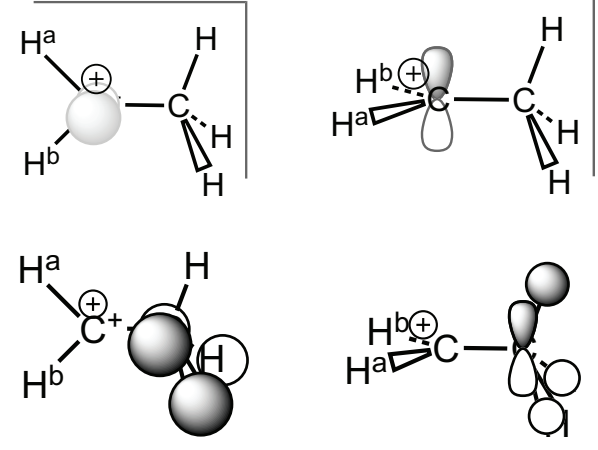

C

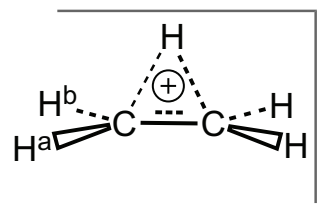

SCHEME 3 | Ethyl carbocation, conformations and corresponding hyperconjugated orbitals. (A) Cs conformation 1a with hyperconjugation from a $\pi-\mathrm{CH}$ bonding orbital; (B) $\mathrm{Cs}$ conformation $1 \mathrm{~b}$ with hyperconjugation from a $\sigma-\mathrm{CH}$ bonding orbital; (C) $\mathrm{C}_{2 v} \mathrm{H}^{+}$bridged conformation 1c.

orbital (Scheme 3B) is a minimum at the Hartree-Fock level, but this minimum collapses to a bridged conformation (Scheme 3C) at correlated levels of calculation such as B3LYP, and CCSD for both $6-311 \mathrm{G}(\mathrm{d})$ and cc-pvQZ basis sets. The corresponding HF/6-311G(d) optimized geometries are displayed in Figure 2 and both energetics and geometrical values are in Table 1 . The results with the cc-pvQZ basis set are given in the supplementary materials.

Because it involves bond breaking/forming, the bridged cation (1c) needs a priori a higher level of computation than the hyperconjugated system (1a) (van Alem et al., 1998). However, all the correlated levels converged to similar energy differences, within a few $\mathrm{kcal} / \mathrm{mol}$. The average energy difference between the two conformations is $\Delta \mathrm{E}_{\mathrm{ac}}=-4 \pm 2 \mathrm{kcal} / \mathrm{mol}$. This is one order of magnitude smaller that the hyperconjugation energies at work (vide infra).

At the HF/6-311G(d) level the (1b) conformation and the bridged one (1c) are two different minima, but the small $L_{\mathrm{CCH}}$ angle $\left(95^{\circ}\right)$ indicates that the proton transfer has already started in (1b), and is effective in (1c). Such 1,2 transfers are related to chemical reactivity (Crone and Kirsch, 2008) (bonds are changing) rather than hyperconjugation itself. However, the limit between reactivity and resonance is somehow difficult to define in hyperconjugation because the orbitals that act as donors are $\mathrm{C}-\mathrm{H}$ bonding orbitals, hence single bonds are partly broken, which corresponds (partly) to a chemical reaction. For a fair and transferable/comparable evaluation of the hyperconjugation effects, we decided to use the (1a) conformation, even though it is characterized as a transition state. The fact that at the correlated levels (CCSD and B3LYP) conformation (1b) collapses to (1c) has also motivated our choice. The (1a) conformation corresponds to the interaction between a $\pi-\mathrm{CH}$ bonding (filled) orbital and the pure empty $p$ orbital of the carbocation (Scheme 3A). A similar scheme can be drawn for conformation (1b). The hyperconjugation in conformation (1a) is shown by both the $\mathrm{CC}^{+}$distance, which is shorter than a normal single bond, and the out of plane $\mathrm{CCH}$ angle which is smaller than normal $\mathrm{sp}^{3}$ angles (e.g., at the B3LYP/6-311G(d) level $\mathrm{d}_{\mathrm{CC}^{+}}=1.412 \AA$ and $\angle_{\mathrm{CCH}}=108^{\circ}-$ Table 1). The results are similar with the cc-pvQZ basis (see Supplementaries, Table S1).

\section{RESULTS AND DISCUSSION}

The results for hyperconjugation in simple carbocations are in Table 2 and Figure 3, while Table 3 and Figure 4 concern the evaluation of the hyperconjugation in conjugated carbocations. In the tables, the two first columns correspond to VRE and ARE as defined in Figure 1. In the three last columns is the $\mathrm{CC}^{+}$ bond variation when hyperconjugation is activated. The $\mathrm{CC}^{+}$ bond shortens when the delocalization is allowed and $\Delta \mathrm{d}$ is thus always negative. These results were of course expected since the electronic delocalization evidently builds a kind of $\pi$ bonding between these two atoms. In the discussions that follow, VRE's are used more often than ARE's because their definition is more straightforward.

To have in mind an order of magnitude for our calculations, we shall recall that for the allyl cation, the VRE amounts to $56.0 \mathrm{kcal} / \mathrm{mol}$ (Table 2, entry 7). This value is to be considered as large.

\section{HYPERCONJUGATION IN THE ETHYL CATION}

The HF geometries and energies obtained for the ethyl cation are similar to those obtained previously by Mo with the ODP procedure (Mo, 2006). With the B3LYP approximation there is a shortening of the $\mathrm{CC}^{+}$bond, from $1.438 \AA$ at the HF level, down to $1.412 \AA$. For the energetic values, we expected an increase at the correlated level, just as it was the case for allyl cation. In this case, Mo reported a resonance energy of $36.6 \mathrm{kcal} / \mathrm{mol}$ at the HF/6-311+G(d) level (Mo, 2006), and $48.8 \mathrm{kcal} / \mathrm{mol}$ with B3LYP/6-311+G(d,p) ${ }^{6}$ (Mo et al., 2007), which corresponds to $33 \%$ of increase. The relative variation is larger for the ethyl cation: the ARE varies here from $12.3 \mathrm{kcal} / \mathrm{mol}$ at the HF level (Table 2 entry 1) to 23.2 with B3LYP (entry 2). It corresponds to $90 \%$ of increase. The VRE amounts to nearly $30 \mathrm{kcal} / \mathrm{mol}$. Hyperconjugation is thus smaller than conjugation in the allyl cation, but the order of magnitude is similar, with a ratio ethyl/allyl $=0.53$.

NBO calculations ${ }^{7}$ on the ethyl cation give access to a second order perturbation theory analysis of the Fock matrix, where the hyperconjugation is evaluated to $18.2 \mathrm{kcal} / \mathrm{mol}$ for each of the two $\mathrm{CH}$ bonds concerned. The total hyperconjugation can thus be evaluated to $36 \mathrm{kcal} / \mathrm{mol}$ with this approach, which is slightly larger than our BLW evaluation. The electronic transfer

\footnotetext{
${ }^{6}$ These are Adiabatic Resonance Energies (ARE). The value we report in Table $2(49.1 \mathrm{kcal} / \mathrm{mol})$ is slightly different from Mo's value due to the basis set difference: we used no diffuses on heavy atoms, and no $\mathrm{p}$ orbitals on the Hydrogens.

${ }^{7}$ NBO 5. Glendening, G. E. D., Badenhoop, J. K., Reed, A. E., Carpenter, J. E., Bohmann, J. A., Morales, C. M., et al. (Theoretical Chemistry Institute, University of Wisconsin, Madison, WI, 2004). Available online at: http://www.chem.wisc.edu/ nbo5
} 
Table 2 | Hyperconjugated carbocations: vertical and adiabatic resonance energy (VRE and ARE) for the hyperconjugated systems.

\begin{tabular}{|c|c|c|c|c|c|c|}
\hline \multicolumn{2}{|c|}{ Species } & $\begin{array}{l}\text { VRE } \\
19.6\end{array}$ & $\begin{array}{r}\text { ARE } \\
12.3\end{array}$ & $\frac{\mathbf{d}_{\mathbf{C c}^{+}}(\mathbf{d e l o c})}{1.438}$ & $\frac{\mathbf{d}_{\mathbf{C C}^{+}} \text {(loc) }}{1.510}$ & $\begin{array}{c}\Delta \boldsymbol{d}^{\mathrm{a}} \\
-0.07\end{array}$ \\
\hline 1 & $\mathrm{CH}_{3}-\mathrm{CH}_{2+}$ & 29.7 & 23.2 & 1.412 & 1.513 & -0.10 \\
\hline 3 & $\left(\mathrm{CH}_{3}\right)_{3}-\mathrm{C}^{+}$ & 45.0 & 40.7 & 1.463 & 1.517 & -0.05 \\
\hline 4 & ${ }^{i} \mathrm{Pr}-\mathrm{CH}_{2+}$ & 33.0 & 25.6 & 1.409 & 1.529 & -0.12 \\
\hline 7 & $\mathrm{H}_{2} \mathrm{C}=\mathrm{CH}-\mathrm{CH}_{2^{+}}$ & 56.0 & 49.1 & 1.381 & 1.498 & -0.12 \\
\hline
\end{tabular}

B3LYP/6-311G(d) level is used, unless specified. All the structures are Cs symmetric and resemble to structure (a) of the ethyl cation-Scheme $\mathbf{3}$.

${ }^{a} \Delta d=d_{C C^{+}}\left(\right.$deloc) $-d_{C C^{+}}(l o c)$.

${ }^{b} H F / 6-311 G(d)$ level.

${ }^{c} \mathrm{DSM}=-\mathrm{CH}\left(\mathrm{SiH}_{3}\right)_{2}$.

${ }^{d} \mathrm{Si}_{-} \mathrm{C}^{+}$bond.

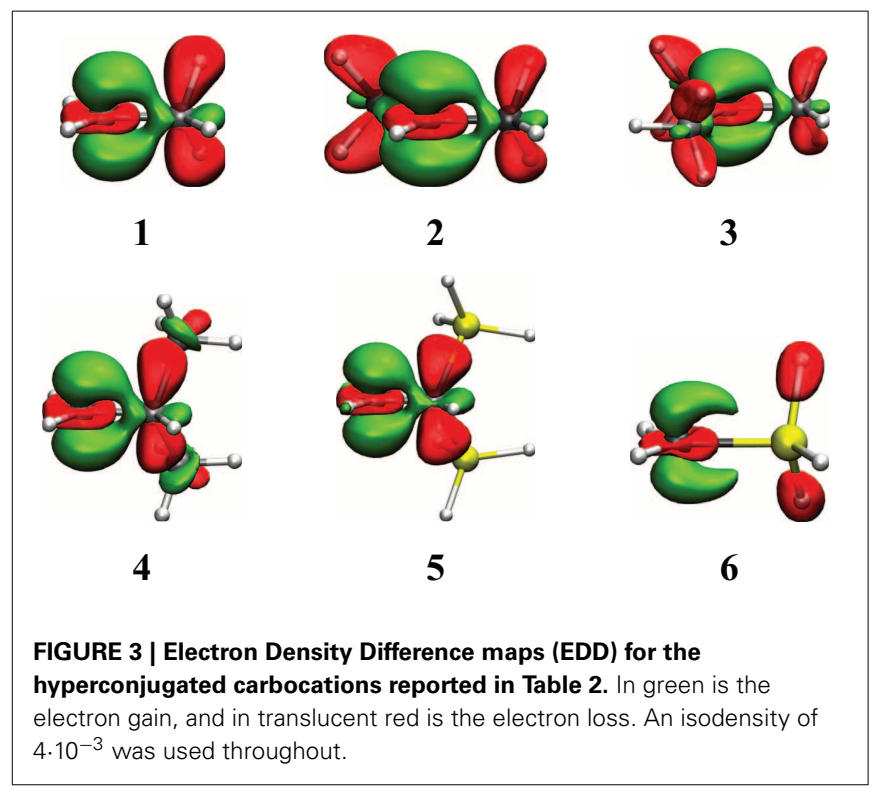

from the $\mathrm{CH}$ bonds to the $\mathrm{C}^{+}$atom amounts to 0.27 electron. For comparison, on the allyl cation NBO gives an interaction between the $\pi$ bond and the $\mathrm{C}^{+}$atom that amounts to $127 \mathrm{kcal} / \mathrm{mol}$. Very logically, this electron transfer concerns 0.5 electron. With the $\mathrm{NBO}$ approach the ratio of the interactions is ethyl/allyl $=0.28$. It is somehow smaller than with BLW. However, the perturbative evaluation of the interaction energy in the allyl might be subject to some caution due to the large effect we are addressing here perturbatively.

Both BLW and NBO evaluations of the hyperconjugation in the ethyl cation give a relatively strong hyperconjugative interaction, and this is consistent with the significant $\mathrm{CC}^{+}$bond shortening, $\Delta d=-0.10 \AA$. We shall note that almost the same shortening is obtained in the allyl (Table 2, $\Delta d=-0.12 \AA$ ).

\section{HYPERCONJUGATION AND SUBSTITUTION EFFECTS}

The substitution effects can be studied via two types of systems, depending on whether the substitution takes place on the carbocation atom, leading to secondary and tertiary carbocations, (cases 1, 2, 3, 6 in Table 2) or if it takes place on the atom at the $\alpha$-position (hence leading to $\beta$-substituted primary carbocations) (cases 4 and 5 ).

The EDD map displayed in Figure 3 shows clearly the electron loss along the two $\mathrm{CH}$ bonds and the electron gain, with the shape of a $\pi$ bond between the two carbon atoms. This corresponds to the idealized picture of hyperconjugation (Scheme 1). These EDD can only be used qualitatively, but very large differences can be visualized. For instance, the delocalization is obviously much larger in the ethyl cation (1) than in the $\mathrm{SiH}_{3}$ substituted equivalent (6). The computed energetics are consistent with the drawing: the VRE amounts to $29.7 \mathrm{kcal} / \mathrm{mol}$ in 1 , but is as small as $12.6 \mathrm{kcal} / \mathrm{mol}$ in $\mathbf{6}$.

For cases 1, 2, 3, not surprisingly, secondary and tertiary carbocations have larger and larger delocalization energy, up to $\mathrm{VRE}=45 \mathrm{kcal} / \mathrm{mol}$ for the tertiary carbocation $\left(\mathrm{CH}_{3}\right)_{3}-\mathrm{C}^{+}$. It is interesting to note that this value is similar to the conjugation in allyl. ${ }^{8}$ The effects of the methyl groups are not additive though. The first methyl brings about $30 \mathrm{kcal} / \mathrm{mol}, 10$ for the second, 5 for the third. Hence, the average stabilization is $15 \mathrm{kcal} / \mathrm{mol}$ per methyl group. In $\left(\mathrm{CH}_{3}\right)_{3} \mathrm{C}-\mathrm{C}^{+}$, the three $\mathrm{CC}^{+}$bond shortenings are accordingly smaller than in the ethyl cation, $\Delta d=$ $-0.05 \AA$ although no direct correlation between bond shortening and hyperconjugation energy can be drawn. Steric effects may also be considered to moderate the bond shortening.

For cases 4 and 5, the substitution with two methyls in $\alpha$ position (4) gives almost no change in the delocalization energy as compared to ethyl cation. It is larger by only $3 \mathrm{kcal} / \mathrm{mol}$ $(\mathrm{VRE}=33.0 \mathrm{kcal} / \mathrm{mol})$. This variation is similar to the variation reported using other approaches, for instance by Aue with the Hydride ion affinity $(+5 \mathrm{kcal} / \mathrm{mol}$ ) (Aue, 2011). The disilyl $\left(\mathrm{SiH}_{3}\right)_{2}$ substitution (5) corresponds to a $\beta$-substituents, and leads to a significant increase in the resonance energy (by almost $+30 \mathrm{kcal} / \mathrm{mol}$ ). It is much larger than for the dimethyl (4) $\left(\mathrm{CH}_{3}\right)_{2}$ moieties. The delocalization energy, VRE $=$ $61.8 \mathrm{kcal} / \mathrm{mol}$, is larger than the resonance energy in the allyl cation (7) at the same level.

\footnotetext{
${ }^{8}$ The ordering is different from the one given by Alabugin et al. (2011). Our values are also systematically larger. We also note that the geometrical relaxation does not explain all the energy difference between Alabugin's and Mo's results (Mo, 2006): correlation effects are important as well.
} 
Table 3 | Vertical and adiabatic resonance energy (VRE and ARE) for the conjugated systems.

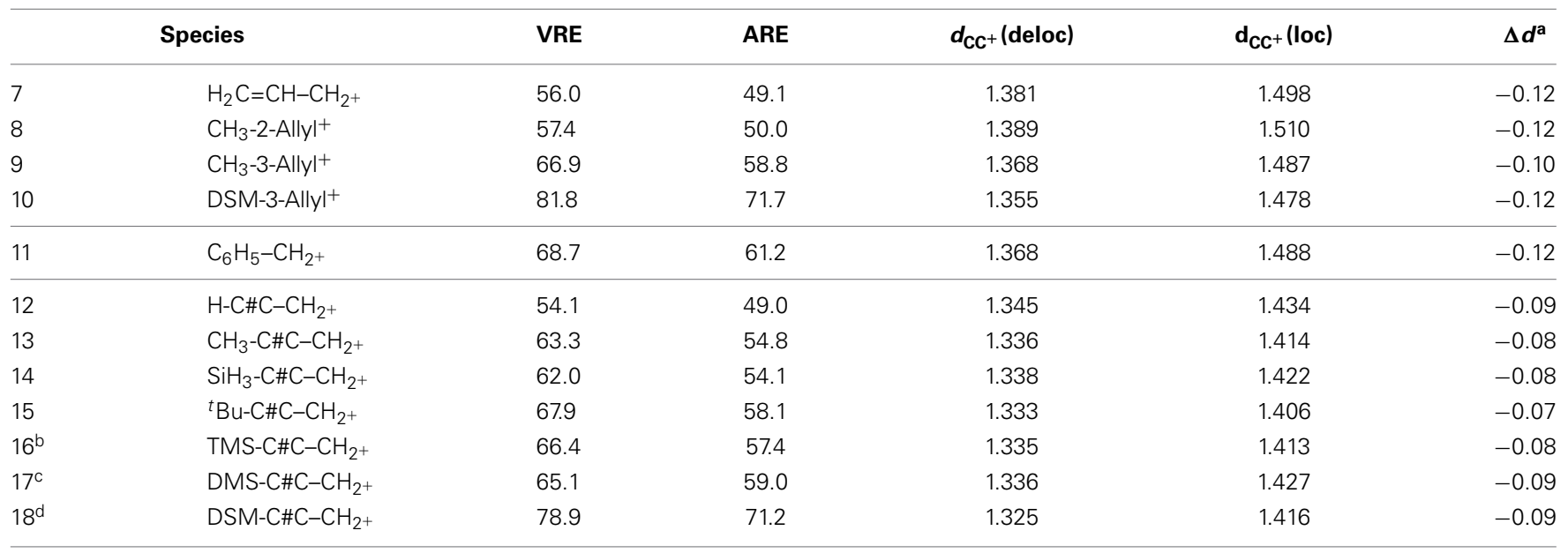

Calculations are at the same level as in Table 2.

${ }^{a} \Delta d=d_{C C^{+}}($deloc $)-d_{C C^{+}}(l o c)$.

${ }^{b} T M S=-S i(M e)_{3}$.

${ }^{c} \mathrm{DMS}=-\mathrm{SiH}(\mathrm{Me})_{2}$.

${ }^{d} \mathrm{DSM}=-\mathrm{CH}\left(\mathrm{SiH}_{3}\right)_{2}$.

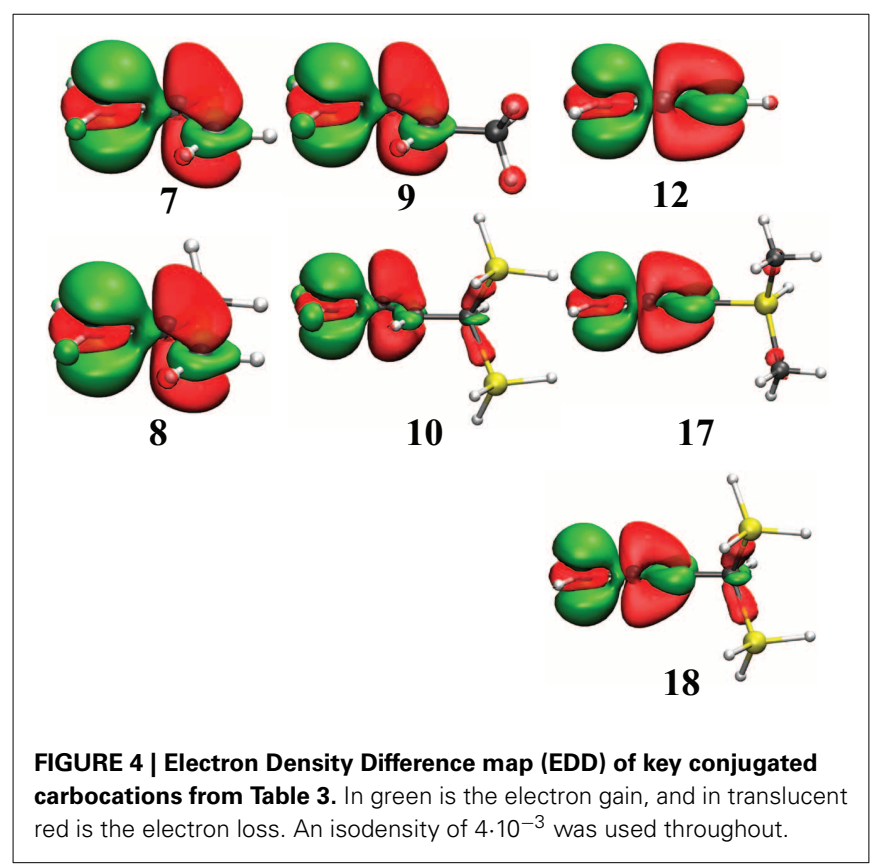

Our results correspond roughly to Frenking's EDA evaluation of the relative stabilization energies between these two systems (33 kcal/mol) (Fernandez and Frenking, 2007), and similar results were reported by others for such silicon in $\beta$-position, for instance by isodesmic reactions ${ }^{9}$ (Lambert, 1990; Creary and Kochly, 2009). The delocalization energy differences are similar, but the delocalization energies differ, sometimes significantly. For instance, Frenking's EDA approach gives almost twice larger $\Delta \mathrm{E}_{\pi}(100 \mathrm{kcal} / \mathrm{mol}$ for the di-silyl substitution) (Fernandez and Frenking, 2007).

\footnotetext{
${ }^{9}$ See for instance Lambert (1990).
}

One shall also note that the bond shortening for the $\mathrm{CC}^{+}$bond is similar for these three primary carbocations, although the resonance energy can be very different. Although it is true that the $\mathrm{CC}^{+}$distance variation reflects hyperconjugation, linear correlations cannot systematically be drawn. ${ }^{10}$ In 6 , the $\mathrm{Si}^{-} \mathrm{C}^{+}$bond distance shortens by about $-0.12 \AA$, which is a large difference for such a small energetic effect. These $\mathrm{SiC}$ bonds are in principle longer and more flexible than CC bonds. ${ }^{11}$ The distance changes upon delocalization $(\Delta d)$ are probably less relevant than the actual bond length, obtained with standard calculations (that is without block localization constraints).

\section{HYPERCONJUGATION IN CONJUGATED SYSTEMS}

We already mentioned that conjugation and hyperconjugation might have similar stabilization energies. We include in this part a few examples where conjugation is evaluated in typical systems such as the already discussed allyl cation, the aromatic benzyl cation and the $\mathrm{C \# C}$ triple bond. These results are extended with some substituted systems to study how additional hyperconjugation operates in already conjugated systems. The results are in Table 3, with some EDD maps in Figure 4.

The hyperconjugation effect on the allyl cation (7) is shown with three substitutions: one on position $2(8)$ and the other two on position $3(\mathbf{9}, \mathbf{1 0})$ (Scheme 4). In 8 there is almost no effect: the resonance energy with the methyl substituent $(57.4 \mathrm{kcal} / \mathrm{mol})$ is very similar to the unsubstituted case $(56.0 \mathrm{kcal} / \mathrm{mol})$, but for a substituent in position 3, the resonance energy increases by about $+10 \mathrm{kcal} / \mathrm{mol}(66.9 \mathrm{kcal} / \mathrm{mol}$ in 9) with a methyl, and by +25 for the di-silyl methyl $(81.8 \mathrm{kcal} / \mathrm{mol}$ in 10$)$. These

\footnotetext{
${ }^{10}$ However, convincing linear regressions have been discussed on the subject, see Fernandez and Frenking (2007).

${ }^{11} v_{\mathrm{CC}}=995 \mathrm{~cm}^{-1}$ vs. $\nu_{\mathrm{SiC}}=700 \mathrm{~cm}^{-1}$, values extracted from http:// webbook.nist.gov (a) $v_{\mathrm{CC}}$ from Shimanouchi (1971) (b) $v_{\mathrm{SiC}}$ from Shimanouchi (1977).
} 
<smiles>C=C(C)C</smiles>

8<smiles>C=CC=O</smiles>

9<smiles>CC([SnH])CSC=O</smiles>

10

SCHEME 4 | Substituted allyl cations.

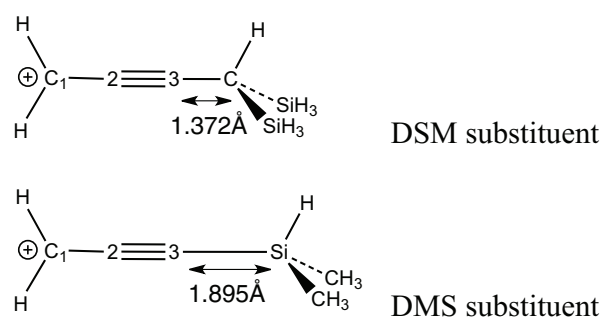

SCHEME 5 | Distance between the hyperconjugated substituent and the "unsaturated" system.

hyperconjugated substituents act exactly as any conjugated $\mathrm{sp}^{3}$ substituent would act (e.g., $-\mathrm{OH},-\mathrm{NH}_{2}$ ). In 8 the methyl is conjugated with the double bond, but it is deconjugated from the positive charge on $\mathrm{C}_{1}$, hence its effect is negligible when delocalization is forbidden/allowed on $\mathrm{C}_{1}$. In $\mathbf{9}$, the methyl is conjugated with both the double bond and with $\mathrm{C}_{1}$, hence the effect that we calculated on $\mathrm{C}_{1}$ is enhanced. It is even one of the largest resonance effects: it is similar to that of the benzyl cation (11), $68.7 \mathrm{kcal} / \mathrm{mol}$.

The delocalization effects in the triply bonded systems are evaluated in the remaining systems (12-18). It is shown on the unsubstituted case that delocalization effects in the propyne cation (12) are similar to the allyl (7). In both species the resonance energy is evaluated to about $55 \pm 1 \mathrm{kcal} / \mathrm{mol}$. Substitutions at the acidic position in these systems increase the stabilization energies by about $+10 \mathrm{kcal} / \mathrm{mol}$ for most species. The substitution by either a methyl (13) or a silyl (14) gives approximately the same resonance energy (about $63 \mathrm{kcal} / \mathrm{mol}$ ). Larger substituents such as ter-Butyl (15), tri-Methyl Silyl (TMS) (16) or di-Methyl Silyl (DMS) (17) leads to only slightly larger resonance energies $(65-68 \mathrm{kcal} / \mathrm{mol})$. However, large resonance energy is found (again) with di-Silyl-Methyl (DSM) derivative. In that case, the (vertical) resonance energy increases to $78.9 \mathrm{kcal} / \mathrm{mol}$. This value corresponds to an increment of some $+25 \mathrm{kcal} / \mathrm{mol}$ (compared to the unsubstituted case), as it was the case for the allyl (10).

The large resonance energy corresponds to more efficient $\sigma$ bond delocalization. However, in both DSM and DMS, the same type of $\mathrm{SiC}$ (or CSi) $\sigma$-bonds interacts with the conjugated carbocation. The stabilization increment is significantly larger for DSM $(+25 \mathrm{kcal} / \mathrm{mol})$ than for DMS $(+10)$ we shall attribute it to the rather short distance between the conjugated link and the CSi bond in DSM (which is much smaller for DSM than
DMS - Scheme 5). The interaction would finally be favored due to a better overlap.

Similarly to the previous series, EDD maps can be used to visualize main electronic effects in these conjugated systems (Figure 4). Of course, most of the delocalization comes from the conjugated link, but larger hyperconjugations correspond to larger domains of electron loss. This is the case for the cations $\mathbf{1 0}$ and 18 but much smaller domains appear for 9 and 17.

\section{CONCLUSION}

Using B3LYP we pinned down resonance energies in a variety of carbocations, with a special attention to hyperconjugative effects. Our discussion focused of vertical resonance energies, and we showed here how hyperconjugation could be of similar magnitude as conjugation, but this evaluation necessitates some correlated methods.

The fact that we considered cationic systems enhanced the delocalization effects. Smaller effects are expected (and reported) for neutral systems (Fernandez and Frenking, 2006). Nevertheless, a dimethyl-silyl substituent (DMS), delocalizes a significant amount of electron density from the CSi bonds onto the neighboring $\mathrm{C}^{+}$, and this hyperconjugation corresponds to a stabilization energy as large as $61.8 \mathrm{kcal} / \mathrm{mol}$. This is to be compared to the vinyl delocalization onto the $\mathrm{C}^{+}$, in the allyl cation. It amounts to "only" $56.0 \mathrm{kcal} / \mathrm{mol}$ of conjugation; hence hyperconjugative effects on energy can be larger than conjugation. The $\mathrm{CC}^{+}$bond distances are accordingly short, e.g., $1.371 \AA$ for the DMS- $\mathrm{CH}_{2}+$ carbocation.

Long-range hyperconjugative effects travel across an unsaturated linkage (a double or a triple bond here). We show that the energy associated to them can be as large as $25 \mathrm{kcal} / \mathrm{mol}$. They can be logically extended to aryl linkages, for instance in para substituted benzyl cations.

\section{SUPPORTING INFORMATION AVAILABLE:}

Cartesian coordinates of all the compounds discussed in the text.

\section{ACKNOWLEDGMENTS}

Prof. Yirong Mo is gratefully acknowledged for the BLW program, and helpful recommendations. Dr. Paola Nava is also gratefully acknowledged for helpful discussions and her pertinent advices.

\section{SUPPLEMENTARY MATERIAL}

The Supplementary Material for this article can be found online at: http://www.frontiersin.org/journal/10.3389/fchem. 2013.00037/abstract 


\section{REFERENCES}

Alabugin, I. V., Gilmore, K. M., and Peterson, P. W. (2011). Hyperconjugation. WIREs Comput. Mol. Sci. 1, 109-141. doi: 10.1002/wcms.6

Aue, D. H. (2011). Carbocations. WIREs Comput. Mol. Sci. 1, 487-508. doi: $10.1002 /$ wcms. 12

Barbour, J. B., and Karty, J. M. (2004). Resonance energies of the allyl cation and allyl anion: contribution by resonance and inductive effects toward the acidity and hydride abstraction enthalpy of propene. J. Org. Chem. 69, 648-654. doi: 10.1021/jo035189m

Becke, A. D. (1993). A new mixing of Hartree-Fock and local density-functional theories. J. Chem. Phys. 98, 1372-1277. doi: 10.1063/1.464304

Bickelhaupt, F. M., and Baerends, E. J. (2003). The case for steric repulsion causing the staggered conformation of ethane. Angew. Chem. 42, 4183-4188. doi: 10.1002/anie.200350947

Braïda, B., Prana, V., and Hiberty, P. C. (2009). The physical origin of Saytzeff's rule. Angew. Chem. Int. Ed. 48, 5724-5728. doi: 10.1002/anie.200901923

Cembran, A., Song, L., Mo, Y., and Gao, J. (2009). Block-localized density functional theory (BLDFT), diabatic coupling, and their use in valence bond theory for representing reactive potential energy surfaces. J. Chem. Theor. Comput. 5, 2702-2716. doi: 10.1021/ct9002898

Cieplak, A. S. (1999). Inductive and resonance effects of substituents on $\pi$-face selection. Chem. Rev. 99, 1265-1336. doi: 10.1021/cr980381n

Creary, X., and Kochly, E. D. (2009). Systematic repression of $\beta$-silyl carbocation stabilization. J. Org. Chem. 74, 2134-2144. Subsequent corrections: 7210-7210. doi: $10.1021 /$ jo802722z

Crone, B., and Kirsch, S. F. (2008). 1, 2-Alkyl migration as a key element in the invention of cascade reactions catalyzed by $\pi$-acids. Chem. Eur. J. 14, 3514-3522. doi: 10.1002/chem.200701985

Dabbagh, H. A., Zamani, M., and Fakhraee, S. (2012). DFT, NBO, and NRT analysis of alkyl and benzyl $\beta$-silyl substituted cations: carbenium ion vs. silylium ion. Res. Chem. Intermed. 38, 1561-1570. doi: 10.1007/s11164-012-0483-3

Dabbagh, H. A., Zamani, M., and Fakhraee, S. (2013). The nature of resonance and hyperconjugation for cyclic $\beta$-silyl substituted carbocations: NBO, NRT, EDA, and NMR studies. Res. Chem. Intermed. 39, 2011-2033. doi: 10.1007/s11164012-0733-4

Daudey, J. P., Trinquier, G., Barthelat, J. C., and Malrieu, J. P. (1980). Decisive role of $\pi$ conjugation in the central bond length shortening of butadiene. Tetrahedron 36, 3399-3401. doi: 10.1016/0040-4020(80)80190-6

Emanuelsson, R., Wallner, A., Ng, E. A. M., Smith, J. R., Nauroozi, D., Ott, S., et al. (2013). $\pi$-conjugated and saturated molecular segments. Angew. Chem. Int. Ed. 52, 983-987. doi: 10.1002/anie.201206030

Feixas, F., Matito, E., Poater, J., and Solà, M. (2011). Understanding conjugation and hyperconjugation from electronic delocalization measures. J. Phys. Chem. A 115, 13104-13113. doi: 10.1021/jp205152n

Fernandez, I., and Frenking, G. (2006). Direct estimate of the strength of conjugation and hyperconjugation by the energy decomposition analysis method. Chem. Eur. J. 12, 3617-3629. doi: 10.1002/chem.200501405

Fernandez, I., and Frenking, G. (2007). Hyperconjugative stabilization in alkyl carbocations: direct estimate of the $\beta$-effect of group 14 elements. J. Phys. Chem. A 111, 8028-8035. doi: 10.1021/jp073737k

Fernandez, I., Wu, J. I., and Schleyer, P. v. R. (2013). Substituent effects on "hyperconjugative" aromaticity and antiaromaticity in planar cyclopolyenes. Org. Lett. 15, 2990-2993. doi: 10.1021/ol401154r

Frisch, M. J., Trucks, G. W., Schlegel, H. B., Scuseria, G. E., Robb, M. A., Cheeseman, J. R., et al. (2004). GAUSSIAN 03. Wallingford, CT: Gaussian, Inc.

Galbraith, J. M., James, A. M., and Nemes, C. T. (2013). The effect of diffuse basis functions on valence bond structural weights. Mol. Phys. 1-7. doi: 10.1080/00268976.2013.850179

Glendening, E. D., Landis, C. R., and Weinhold, F. (2012). Natural bond orbital methods. WIREs Comput. Mol. Sci. 2, 1-42. doi: 10.1002/wcms.51

Gobbi, A., and Frenking, G. (1994). Resonance stabilization in allyl cation, radical, and anion. J. Am. Chem. Soc. 116, 9275-9286. doi: 10.1021/ja00099a052

Hadzic, M., Braïda, B., and Volatron, F. (2011). Wheland intermediates: an ab initio valence bond study. Org. Lett. 13, 1960-1963. doi: 10.1021/ol200327s

Havenith, R. W. A., and Van Lenthe, J. H. (2009). Delocalization in valence bond hyperconjugation. Int. J. Quant. Chem. 109, 2426-2429. doi: 10.1002/qua.22018

Hehre, J. W. (1975). Theoretical approaches to the structure of carbocations. Acc. Chem. Res. 8, 369-375. doi: 10.1021/ar50095a002

Humphrey, W., Dalke, A., and Schulten, K. (1996). VMD-visual molecular dynamics. J. Mol. Graph. 14, 33-38. doi: 10.1016/0263-7855(96)00018-5
Ingold, K. U., and DiLabio, G. A. (2006). Bond strengths: the importance of hyperconjugation. Org. Lett. 8, 5923-5925. doi: 10.1021/ol062293s

Krishnan, R., Binkley, J. S., Seeger, R., and Pople, J. A. (1980). Self-consistent molecular-orbital methods. 20. Basis set for correlated wave-functions. J. Chem. Phys. 72, 650-654. doi: 10.1063/1.438955

Lambert, J. B. (1990). The interaction of silicon with positively charged carbon. Tetrahedron 46, 2677-2689. doi: 10.1016/S0040-4020(01)88362-9

Lambert, J. B., and Chelius, E. C. (1990). $\beta$-Effect of silicon in the synperiplanar geometry. J. Am. Chem. Soc. 112, 8120-8124. doi: 10.1021/ja00178a041

Lambert, J. B., and Ciro, S. M. (1996). The interaction of $\pi$ orbitals with a carbocation over three $\sigma$ bonds. J. Org. Chem. 61, 1940-1945. doi: 10.1021/ jo951643d

Lee, C., Yang, W., and Parr, R. G. (1988). Development of the Colle-Salvetti correlation-energy formula into a functional of the electron density. Phys. Rev. B 37, 785-789. doi: 10.1103/PhysRevB.37.785

Linares, M., Braïda, B., and Humbel, S. (2006). Lewis-based valence bond scheme: application to the allyl cation. J. Phys. Chem. A 110, 2505-2509. doi: 10.1021/jp056090h

Linares, M., Humbel, S., and Braïda, B. (2008). The nature of resonance in allyl ions and radical. J. Phys. Chem. A 112, 13249-13255. doi: 10.1021/jp8038169

McLean, A. D., and Chandler, G. S. (1980). Contracted gaussian-basis sets for molecular calculations.1. 2nd row atoms, $\mathrm{Z}=11-18$. J. Chem. Phys. 72, 5639-5648. doi: 10.1063/1.438980

Milian-Medina, B., and Gierschner, J. (2012). $\pi$-conjugation. WIREs Comput. Mol. Sci. 2, 513-524. doi: 10.1002/wcms. 95

Mo, Y. (2004). Resonance effect in the allyl cation and anion: a revisit. J. Org. Chem. 69, 5563-5567. doi: 10.1021/jo049822v

Mo, Y. (2006). Intramolecular electron transfer: computational study based on the orbital deletion procedure (ODP). Curr. Org. Chem. 10, 779-790. doi: $10.2174 / 138527206776818946$

Mo, Y., Bao, P., and Gao, J. (2011). Energy decomposition analysis based on a blocklocalized wavefunction and multistate density functional theory. Phys. Chem. Chem. Phys. 13, 6760-6775. doi: 10.1039/c0cp02206c

Mo, Y., Gao, J., and Peyerimhoff, S. D. (2000). Energy decomposition analysis of intermolecular interactions using a block-localized wavefunction approach. J. Chem. Phys. 112, 5530-5538. doi: 10.1063/1.481185

Mo, Y., Hiberty, P. C., and Schleyer, P. v. R. (2010). How to properly compute the resonance energy within the ab initio valence bond theory: a response to the ZHJVL paper. Theor. Chem. Acc. 127, 27-38. doi: 10.1007/s00214010-0794-7

Mo, Y., Lin, Z., Wu, W., and Zhang, Q. (1996). Delocalization in allyl cation, radical, and anion. J. Phys. Chem. 100, 6469-6474. doi: 10.1021/jp9526612

Mo, Y., and Peyerimhoff, S. (1998). Theoretical analysis of electronic delocalization. J. Chem. Phys. 109, 1687-1696. doi: 10.1063/1.476742

Mo, Y., and Schleyer, P. v. R. (2006). An energetic measure of aromaticity and antiaromaticity based on the Pauling-Wheland resonance energies. Chem. Eur. J. 12, 2009-2020. doi: 10.1002/chem.200500376

Mo, Y., Song, L., and Lin, Y. (2007). Block-localized wavefunction (BLW) method at the density functional theory (DFT) level. J. Phys. Chem. A 111, 8291-8301. doi: 10.1021/jp0724065

Mo, Y., Song, L., Wu, W., and Zhang, Q. (2004a). Charge transfer in the electron donor-acceptor complex $\mathrm{BH}_{3} \mathrm{NH}_{3}$. J. Am. Chem. Soc. 126, 3974-3982. doi: $10.1021 /$ ja0397781

Mo, Y., Wu, W., Song, L., Lin, M., Zhang, Q., and Gao, J. (2004b). The magnitude of hyperconjugation in ethane: a perspective from $\mathrm{Ab}$ initio valence bond theory. Angew. Chem. Int. Ed. 43, 1986-1990. doi: 10.1002/anie.200352931

Müller, T., Margraf, D., and Syha, Y. (2005). $\sigma$-delocalization versus $\pi$-resonance in $\alpha$-aryl-substituted vinyl cations. J. Am. Chem. Soc. 127, 10852-10860. doi: $10.1021 / \mathrm{ja} 0516864$

Mullins, J. J. (2012). Hyperconjugation: a more coherent approach. J. Chem. Educ. 89, 834-836. doi: 10.1021/ed1011986

Newhouse, T., and Baran, P. S. (2011). If C-H bonds could talk: selective C-H bond oxidation. Angew. Chem. Int. Ed. 50, 3362-3374. doi: 10.1002/anie.201006368

Olah, G. A. (1993). Superelectrophiles. Angew. Chem. Int. Ed. Eng. 32, 767-788. doi: 10.1002/anie.199307673

Olah, G. A. (2001). 100 years of carbocations and their significance in chemistry. J. Org. Chem. 5943-5957. doi: 10.1021/jo010438x

Schmidt, M. W., Baldridge, K. K., Boatz, J. A., Elbert, S. T., Gordon, M. S., Jensen, J. H., et al. (1993). General atomic and molecular electronic structure system. J. Comput. Chem. 14, 1347-1363. doi: 10.1002/jcc.540141112 
Schormann, M., Garratt, S., Hughes, D. L., Green, J. C., and Bochmann, M. (2002). Isolation and structure of $\left[\mathrm{HC}\left\{\mathrm{CH}\left(\mathrm{SiMe}_{3}\right)\left(\mathrm{SnMe}_{3}\right)\right\}_{2}\right]^{+}$: a remarkably stable sec-alkyl cation. J. Am. Chem. Soc. 124, 11266-11267. doi: 10.1021/ja026443f

Shaik, S. S., and Hiberty, P. C. (2008). A Chemist's Guide to Valence Bond Theory. New York, NY: Wiley-Interscience.

Shimanouchi, T. (1971). Tables of molecular vibrational frequencies consolidated volume I. Natl. Bur. Stand. 1, 1-160.

Shimanouchi, T. (1977). Tables of molecular vibrational frequencies consolidated volume II. J. Phys. Chem. Ref. Data 6, 993-1102. doi: 10.1063/1.555560

Steinmann, S. N., Mo, Y., and Corminboeuf, C. (2011). How do electron localization functions describe $\pi$-electron delocalization? Phys. Chem. Chem. Phys. 13, 20584-20592. doi: 10.1039/c1cp21055f

van Alem, K., Sudhölter, E. J. R., and Zuilhof, H. (1998). Quantum chemical calculations on r-substituted ethyl cations: a comparison between B3LYP and Post-HF methods. J. Phys. Chem. A 102, 10860-10868. doi: 10.1021/jp983114u

Vosko, S. H., Wilk, L., and Nusair, M. (1980). Accurate spin-dependent electron liquid correlation energies for local spin density calculations: a critical analysis. Can. J. Phys. 58, 1200-1211. doi: 10.1139/p80-159

Weinhold, F. (2003). Rebuttal to the Bickelhaupt-Baerends case for steric repulsion causing the staggered conformation of ethane. Angew. Chemie Int. Ed. 42, 4188-4194. doi: 10.1002/anie.200351777

White, E. T., Tang, T., and Oka, J. (1999). $\mathrm{CH}_{5}^{+}$: the infrared spectrum observed. Science 184, 135-137. doi: 10.1126/science.284.5411.135

Wiberg, K. B., Breneman, C. M., and Lepage, T. J. (1990). Resonance interactions in acyclic systems.1. Energies and charge distributions in allyl anions and related compounds. J. Am. Chem. Soc. 112, 61-72. doi: 10.1021/ja00157a011

Wu, J. I., Fernandez, I., Mo, Y., and Schleyer, P. v. R. (2012). Why cyclooctatetraene is highly stabilized: the importance of "Two- Way" (Double) hyperconjugation. J. Chem. Theory Comput. 8, 1280-1287. doi: 10.1021/ct3000553
Wu, J. I., and Schleyer, P. v. R. (2013). Hyperconjugation in hydrocarbons: not just a "mild sort of conjugation." Pure Appl. Chem. 85, 921-940. doi: 10.1351/PACCON-13-01-03

Zielinski, M., Havenith, R. W. A., Jenneskens, L. W., and Van Lenthe, J. H. (2010). A comparison of approaches to estimate the resonance energy. Theor. Chem. Acc. 127, 19-25. doi: 10.1007/s00214-010-0793-8

Zimmerman, H. E., and Weinhold, F. (2013). Natural bond-bond polarizability: a Hückel-like electronic delocalization index. J. Org. Chem. 78, 1844-1850. doi: $10.1021 /$ jo301620k

Conflict of Interest Statement: The authors declare that the research was conducted in the absence of any commercial or financial relationships that could be construed as a potential conflict of interest.

Received: 04 October 2013; paper pending published: 21 November 2013; accepted: 11 December 2013; published online: 07 January 2014.

Citation: Alamiddine $Z$ and Humbel S (2014) Hyperconjugation in Carbocations, a BLW Study with DFT approximation. Front. Chem. 1:37. doi: 10.3389/fchem. 2013.00037

This article was submitted to Theoretical and Computational Chemistry, a section of the journal Frontiers in Chemistry.

Copyright (c) 2014 Alamiddine and Humbel. This is an open-access article distributed under the terms of the Creative Commons Attribution License (CC BY). The use, distribution or reproduction in other forums is permitted, provided the original author(s) or licensor are credited and that the original publication in this journal is cited, in accordance with accepted academic practice. No use, distribution or reproduction is permitted which does not comply with these terms. 